\title{
Scope, Focus, and Relevance of Ophthalmology Grand Rounds
}

\author{
Evan D. Schoenberg, MD ${ }^{1}$ John Heffernan, MD ${ }^{1}$ David Zeng, MD ${ }^{1}$ \\ Ramesh S. Ayyala, MD, FRCS, FRCOphth ${ }^{1}$ \\ ${ }^{1}$ Department of Ophthalmology, Tulane School of Medicine, Tulane \\ University, New Orleans, Louisiana \\ Journal of Academic Ophthalmology 2014;7:e13-e16.

\begin{abstract}
Address for correspondence Ramesh S. Ayyala, MD, FRCS, FRCOphth, Glaucoma Service, Department of Ophthalmology, Tulane University Medical Center, 1430 Tulane Avenue, SL-69, New Orleans, LA 70112 (e-mail: rayyala@tulane.edu).
\end{abstract}

\begin{abstract}
Purpose The aim of the study is to analyze the scope, focus, and educational relevance of resident grand presentations in relation to the community prevalence and impact of the diseases discussed.

Design This study is a retrospective review of grand rounds (GR) presentations at Tulane University School of Medicine's Department of Ophthalmology over a 3-year period.

Methods The topic of each GR presentation between 2009 and 2012 was categorized by subject matter, prevalence, and impact in a retrospective review of saved PowerPoint files and surgical videos.

Results Two hundred and eighty-four presentations were analyzed. Retina, glaucoma, cornea and external disease, and neuro-ophthalmology each comprised approximately one-sixth (17\%) of topics discussed. Cataract and oculoplastics each comprised $9 \%$, with the remaining cases distributed among other subjects. Sixty-five percent of discussed diseases were rare, while $19 \%$ were uncommon and $15 \%$ were common. Seventy-eight percent of diagnoses had impact on life $(30 \%)$ or vision $(48 \%)$. While diseases with minimal impact were distributed fairly evenly across prevalence categories, $64 \%$ of vision-threatening and $79 \%$ of life-threatening diseases were rare.

Conclusions Our cohort of residents spontaneously divided their topics across most subjects within ophthalmology fairly evenly. Rare diseases comprised a majority of the GR discussions, so the average GR session did not address diagnoses likely seen in an

Keywords

- grand rounds

- resident education

- clinical relevance average clinic. However, these rare diseases were much more likely to be vision- or life threatening. Encouraging a greater emphasis on more commonly encountered pathology could improve GR's daily clinical relevance for resident education, but care must be taken to continue discussion of rare diseases of critical importance.
\end{abstract}

Grand rounds (GR) is a medical education tradition dating back to bedside teaching of the late 19th century. Popularized by clinicians like Sir William Osler long before the advent of PowerPoint presentations, GR was a patient-centered conference, and as attendance outgrew the patient's own room, the "rounds" were held in an amphitheater with the patient as invited guest. ${ }^{1}$
GR means different things in different fields and at different learning centers. Medical grand rounds (MGR) is well described in the literature. A 2006 Mayo Clinic survey of the departments of medicine in U.S. medical schools by Mueller et al, based in part on a similar 1988 survey by Parrino and White, characterized MGR as a weekly (per 96\% of respondents) hour-long (98\%) didactic lecture (95\%) on a
DOI http://dx.doi.org/ 10.1055/s-0034-1396087. ISSN 2164-7879.
Copyright $\odot 2014$ by Thieme Medical Publishers, Inc., 333 Seventh Avenue, New York, NY 10001, USA. Tel: +1(212) 584-4662.
License terms

()(1) $\Theta \circledast$ 
single topic (98\%), typically chosen by the presenter (67\%) and based around a case presentation less than half the time (58\%). GR is offered by $97 \%$ of academic medical departments, with patients present less than $3 \%$ of the time. Top priorities of MGR were reported to be providing updates in diagnosis and treatment, providing updates in medical research, educating internal medicine house staff, and educating faculty. ${ }^{2}$

Ophthalmology grand rounds (OGR), on the other hand, is sparsely explored. At our institution, Tulane University in New Orleans, Louisiana, OGR is a weekly 2-hour conference in which three cases are presented. One first-year and one second-year resident each present a PowerPoint-based didactic lecture structured around a clinical case. The case is expected to frame discussion of presentation, diagnosis, workup, and management, with varying integration of basic sciences. Residents are encouraged but not required to engage the resident and faculty audience throughout the case. One third-year resident presents a surgical video, discussing challenges and techniques involved in the surgery. After each presentation, the department chairman presides over the assembled faculty questioning the presenter and discussing the case. Resident presenters are responsible for their topic selection, without reference to a needs assessment or a list of assigned topics.

As GR is often considered the "most important" departmental conference, its quality has a direct impact on the quality of trainee education. ${ }^{2}$ It has been suggested that, in the general case of MGR, topic selection based upon needs assessment would positively impact training. ${ }^{3}$ To our knowledge, the current work represents the first analysis of the topics and scope of OGR across a resident cohort. Besides breadth of topics presented, we also analyze and discuss the impact of the diseases discussed.

\section{Methods}

From July 2009 to June 2012, the tenure of the ophthalmology residency class of 2012, all presenting residents at our institution were asked to save their GR videos and PowerPoint presentations to a designated archive (meditred.com). Two hundred and eighty-four presentations in all were archived, and all were included in this retrospective review. As the present study evaluated anonymous, previously collected data, it was determined to be exempt from Institutional Review Board (IRB) review per the standards of the Tulane University IRB after appropriate evaluation. For each presentation, the primary topic of discussion, typically a disease process, was noted. Two ophthalmologists-one senior resident (EDS) and one attending physician (RAS)-categorized each presentation by subspecialty topic, by prevalence of the disease process discussed, and by impact of that disease process. Each grader analyzed the data independently, and any discrepancy between their scores was resolved by reference to clinical textbooks and medical literature.

Prevalence was graded "common," "uncommon," or "rare" based on the frequency of an ophthalmology resident examining a patient with the disease in a comprehensive ophthalmology clinic setting in or around New Orleans, Louisiana. Diseases labeled "common" were estimated to be seen by a resident approximately once per week or more, "uncommon" at least once per three months, and "rare" once to twice per year or less. Examples of "common" diseases discussed in the evaluated GR presentations are dry eye syndrome, cataract, and macular degeneration. Examples of "uncommon" diseases are corneal graft rejection, neovascular glaucoma, and pseudotumor cerebri. Examples of "rare" diseases are aniridia, neurofibromatosis, and orbital aspergillosis. For the purposes of statistical analysis, this scale was recorded as a value between 1 and 3, with 1 being "common" and 3 being "rare."

Impact was graded "minimal/academic interest," "visionthreatening," or "life-threatening" based on the natural history of the disease process. An impact of "minimal" or "academic interest" was assigned if the missing, mismanaging, or delaying diagnosis of the disease would have little long-term impact on outcomes. An impact of "visionthreatening" was assigned if the disease could lead to permanent visual sequelae. Finally, an impact of "life-threatening" was assigned if the disease could lead to serious systemic complications. Examples of "minimal/academic interest" diseases discussed in the evaluated GR presentations are epithelial inclusion cyst, conjunctival nevus, and multiple evanescent white dot syndrome. Examples of "vision-threatening" diseases are macular edema, herpetic stromal keratitis, and nonarteritic anterior ischemic optic neuropathy. Examples of "life-threatening" diseases are new bilateral visual field deficits, neonatal gonococcal conjunctivitis, and cavernous sinus thrombosis. For the purposes of statistical analysis, this scale was recorded as a value between 1 and 3 , with 1 being "minimal" and 3 being "life-threatening."

Statistical analysis was performed in Microsoft Excel.

\section{Results}

A total of 284 presentations from 3 years of GR presentations were analyzed, corresponding with a cycle of one class of residents through the program. During that time, an average of 102 presentations per year were given, for a total of 306 presentations. Twenty-two presentations were not available to be included in analysis because the presenter did not store their talk or video in the designated archive. Overall, $92.8 \%$ of presentations were available for analysis.

Inter-rater agreement for topic and category was $100 \%$. Inter-rater agreement for impact was $100 \%$. Initial inter-rater agreement for prevalence was $95.8 \%$ ( $n=12$ disagreements; Cohen $k=0.933$, consistent with excellent agreement). All disagreements on prevalence were settled to the satisfaction of both raters after reference to the literature.

Ophthalmology is commonly divided into 10 clinical subspecialties (cornea and external disease, glaucoma, pediatric ophthalmology and strabismus, lens and cataract, neuro-ophthalmology, orbit and oculoplastics, refractive surgery, retina and vitreous, and uveitis) plus ophthalmic pathology. Retina, glaucoma, cornea and external disease, and neuro-ophthalmology each comprised approximately one-sixth (17\%) of topics discussed. Cataract and oculoplastics each comprised $9 \%$, with the remaining cases distributed among strabismus, uveitis, and refractive surgery (-Fig. 1). Sixty-five percent of diagnoses discussed represented rare diseases, while $19 \%$ were 


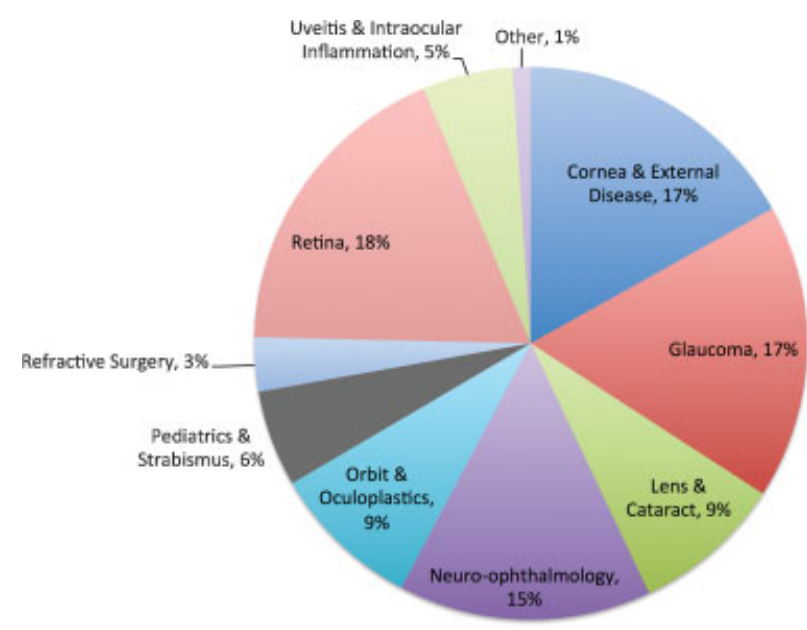

Fig. 1 Tulane grand rounds (GR) presentations categorization. Results of the categorization of GR presentations. Retina (18\%), corneal and external disease (17\%), glaucoma (17\%), and neuro-ophthalmology (15\%) were the most commonly represented topics. $N=284$.

uncommon and $15 \%$ were common (-Fig. 2). Thirty percent of all disease processes, if missed or mismanaged, could be life threatening, and $48 \%$ would be vision threatening. In total, $78 \%$ of all diagnoses had impact on life or vision. While diseases with minimal impact were distributed fairly evenly across prevalence categories, $64 \%$ of vision-threatening and $79 \%$ of life-threatening diseases were rare (-Fig. $\mathbf{3}$ ).

\section{Discussion}

The main purpose of GR is to discuss a clinical case in sufficient detail and analyze its management in an academic learning environment (in the presence of all residents and faculty). The resident presenter typically

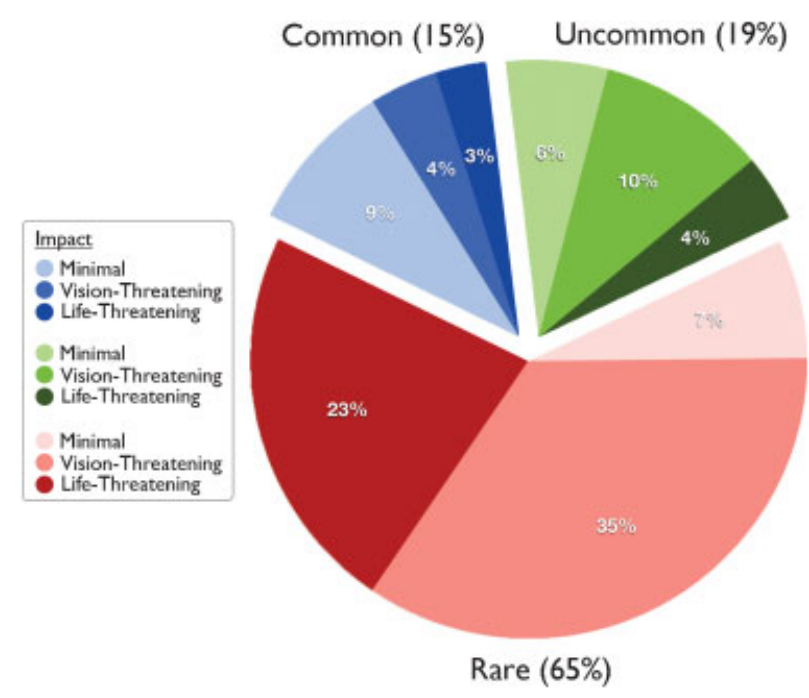

Fig. 2 Prevalence and impact of grand rounds (GR) presentations. GR presentations were predominately on "rare" (65\%) diseases. Diseases labeled "common" are seen approximately once per week, "uncommon" one to three times per month, and "rare" one to two times per year or less in New Orleans.

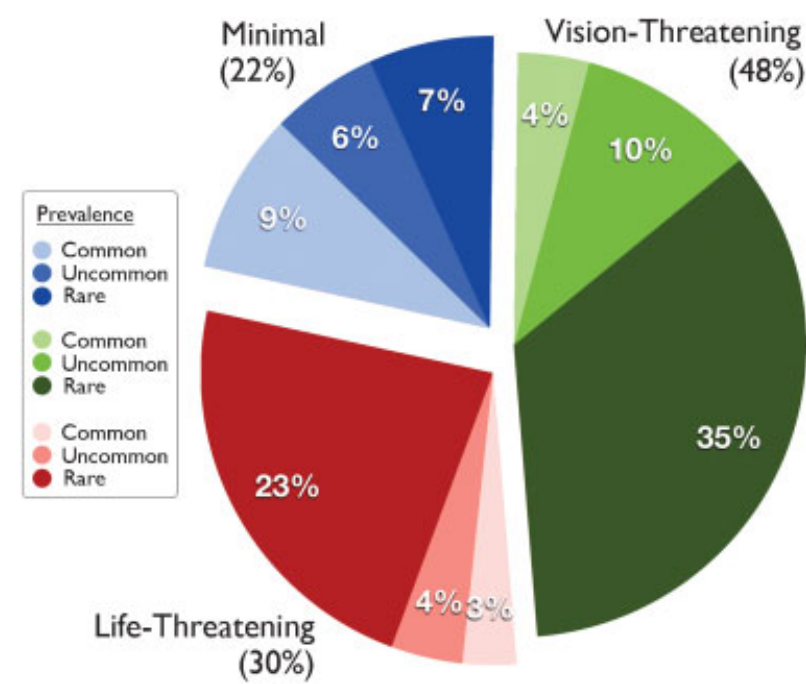

Fig. 3 Impact and prevalence of grand rounds (GR) topics. GR presentations placed a heavy emphasis on life-threatening (30\%) and vision-threatening (48\%) diseases.

has participated in the actual patient care, spent time reviewing the chart, collected and analyzed all the diagnostics tests and management, and read the up-to-date literature on the topic. It is expected that the presenter is able to present the case summary and relevant literature in 10 to 15 minutes. When done properly, GR can satisfy several of the core competency requirements by the ACGME, including patient care, medical knowledge, practice-based learning, systems-based practice, and interpersonal skills and communications. Systematic analysis of GR from a given program might give an insight as to the contribution of GR to resident education.

The choice of GR topic is a key factor in their relevance. Needs assessment and assigned topics are used in 44 and 33\%, respectively, of MGR. ${ }^{4}$ In the Tulane OGR, resident selection alone determined the topics. The cohort of residents at Tulane spontaneously divided their topics across most subjects within ophthalmology relatively evenly, although some underrepresentation occurred in optics, strabismus, uveitis, and refractive surgery. This suggests that assignment may not be necessary in all cases.

Rare diseases comprised a majority of the GR discussions, so the typical GR session did not address diagnoses likely to be seen in an average clinic. These rare diseases, however, were much more likely to be vision- or life-threatening in nature. Thus, these diagnoses, while perhaps encountered only a few times in the typical clinician's practice, represent diagnoses of critical importance. GR could well be the only exposure residents have to the clinical presentation of these rare diseases before encountering them in practice.

Resident fascination with rare cases is a well-known issue in academic circles. It is not uncommon for even medical students to lean toward presenting the unusual case compared with the common diseases that are seen every day in the clinic. The impact of this understandable fascination on the evolution of these young residents' clinical knowledge and on their future clinical practices needs further studies. 
e16 Scope, Focus, and Relevance of Ophthalmology Grand Rounds Schoenberg et al.

The predominance of unusual diseases presented in GR is not inherently misguided. Indeed, one of the purposes of GR is to expose residents to a spectrum of cases they are unlikely to see on their own in clinic, and therefore prepare them to accurately diagnose unusual vision- or life-threatening diseases in their future practice. Yet GR should also serve as a didactic device for the most common topics in ophthalmology, to ensure resident exposure and mastery of the subtler details of typical diseases and surgeries in clinical practice. Encouraging a greater emphasis on common and uncommon diseases could provide greater day-to-day clinical relevance for resident education, but care must be taken to continue discussion of rare diseases that are vision- or life-threatening in nature.

Residency programs could ensure an appropriate variety of GR case presentations by tracking and categorizing the presentations by prevalence and impact following a similar method to ours. Of course, simply presenting a topic does not ensure that successful education has occurred. Future research could assess whether GR ophthalmology case presentations are an optimal teaching method for the adult learners in residency programs.

\section{Conflict of Interest}

Evan D. Schoenberg, John Heffernan, and David Zeng declare no financial interest.
Ramesh S. Ayyala, president at MediTred, has financial interest in the company, which was used for storage and retrieval of grand rounds presentations analyzed in this study.

\section{Funding}

This research had no funding or commercial support.

\section{Access to Data}

The principal investigator had full access to all the data in the study and takes responsibility for the integrity of the data and the accuracy of the data analysis.

\section{References}

1 Bogdonoff MD. A brief look at medical grand rounds. Pharos Alpha Omega Alpha Honor Med Soc 1982;45(1):16-18

2 Hebert RS, Wright SM. Re-examining the value of medical grand rounds. Acad Med 2003;78(12):1248-1252

3 Van Hoof TJ, Monson RJ, Majdalany GT, Giannotti TE, Meehan TP. Improving medical grand rounds: recommendations. Conn Med 2009;73(10):601-607

4 Mueller PS, Segovis CM, Litin SC, Habermann TM, Parrino TA. Current status of medical grand rounds in departments of medicine at US medical schools. Mayo Clin Proc 2006;81(3):313-321 